\title{
Use of a trans-tracheal rapid insufflation of oxygen device in a "cannot intubate, cannot oxygenate" scenario in a parturient -a case report-
}

\author{
John Lee ${ }^{1}$, Von Vee $\mathrm{Ng}^{1}$, Constance $\mathrm{Teo}^{2}$, and Patrick Wong ${ }^{1}$ \\ Departments of ${ }^{1}$ Anesthesiology and ${ }^{2}$ Otolaryngology, Singapore General Hospital, Singapore
}

Background: The trans-tracheal rapid insufflation of oxygen (TRIO) device is less commonly used and is an alternative to trans-tracheal jet ventilation for maintaining oxygenation in a "cannot intubate, cannot oxygenate" (CICO) scenario. Case: We report the successful use of this device to maintain oxygenation after jet ventilator failure in a parturient who presented with the CICO scenario during the procedure for excision of laryngeal papilloma.

Conclusions: A stepwise approach to the airway plan and preparation for an event of failure is essential for good materno-fetal outcomes. The TRIO device may result in inadequate ventilation that can lead to hypercarbia and respiratory acidosis. Hence, it should only be used as a temporizing measure before a definitive airway can be secured.

Keywords: Airway management; Airway obstruction; High-frequency jet ventilation; Insufflation; Obstetrical anesthesia; Trans-tracheal rapid insufflation of oxygen (TRIO).

Trans-tracheal jet ventilation is an established temporizing method of oxygenation in a "cannot intubate, cannot oxygenate" (CICO) scenario. However, trans-tracheal rapid insufflation of oxygen (TRIO) through a cricothyroidotomy cannula is a less familiar yet effective alternative and is readily available in Singapore General Hospital [1]. We describe its successful use in an emergency situation, for maintaining oxygenation after

Corresponding author: John Lee, MBBS, MMed

Department of Women's Anesthesia, KK Women's and Children's Hospital, 100 Bukit Timah Road, Singapore 229899, Singapore

Tel: +65-6394-1081

Email: john.lee.s.e@singhealth.com.sg

ORCID: https://orcid.org/0000-0001-5698-3913

Received: December 3, 2018.

Revised: January 31, 2019.

Accepted: February 16, 2019.

Korean J Anesthesiol 2019 August 72(4): 381-384

https://doi.org/10.4097/kja.d.18.00334 unexpected jet ventilator malfunction in a CICO scenario in a parturient.

\section{Case Report}

A 35-year-old parturient, at $13+1$ weeks of gestation, was scheduled for an elective surgical excision of a recurrent laryngeal papilloma (Fig. 1). In the preoperative clinic, flexible nasoendoscopy showed large papillomatous lesions obscuring the glottic inlet. The patient was breathing well, albeit with mild stridor. She was strongly advised to undergo an awake tracheostomy under local anesthesia, as it was deemed the safest option. However, she adamantly refused as she was extremely anxious, and reported that she had previously undergone a similar operation without the need for a tracheostomy. An awake rigid Bonfils (Karl Storz GmbH \& Co. KG, Germany) fiberoptic intubation was previously used to secure the airway. Using this technique again was considered unsuitable due to the extent of the laryngeal lesions. The following alternative strategy, which

(c) This is an open-access article distributed under the terms of the Creative Commons Attribution Non-Commercial License (http://creativecommons.org/ licenses/by-nc/4.0/), which permits unrestricted non-commercial use, distribution, and reproduction in any medium, provided the original work is properly cited. 


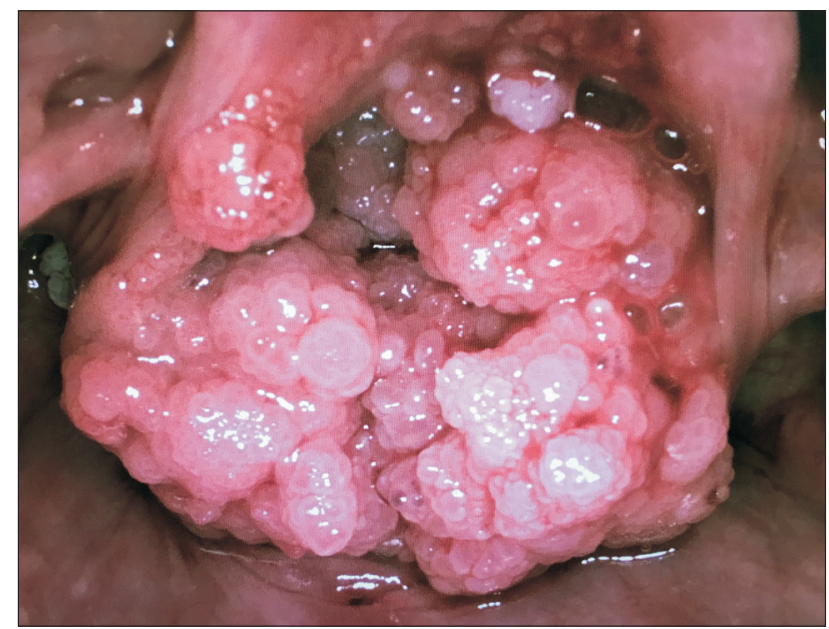

Fig. 1. Laryngeal papilloma.

she agreed to, was offered: plan A - inhalational induction and oral Bonfils intubation; plan B - oxygenation by jet ventilation via needle cricothyroidotomy as a temporizing measure; and, plan C - asleep tracheostomy.

One week later, on the day of surgery, all airway equipment was checked including the TRIO device (Fig. 2), which is our institution's version of the Rapid- $\mathrm{O}_{2}{ }^{\mathrm{TM}}$ (Meditech Systems, Ltd., UK) [1]. The patient's neck anatomy was marked out, and a senior consultant ENT surgeon was scrubbed and available to perform an emergency tracheostomy, should the need arise.

Standard anesthetic monitoring was applied, and her $\mathrm{SpO}_{2}$ was $95 \%$ in room air. Inhalational induction was performed with sevoflurane $6 \%$ in $100 \%$ oxygen. At 2.0 MAC (minimum alveolar concentration), the patient still exhibited spontaneous arm movements. At 2.2 MAC (10 min after the start of induction), she developed severe airway obstruction with a depressed capnography waveform, but her $\mathrm{SpO}_{2}$ remained at $100 \%$. We proceeded with plan B, i.e., Ravussin cannula (VBM Medizintechnik GmbH, Germany) cricothyroidotomy whilst maintaining face mask oxygenation. She then developed complete airway obstruction with absent capnography waveform accompanied by paradoxical chest/abdominal movement after we successfully obtained front of neck access. This was initially relieved by face mask ventilation but became progressively difficult to manage. In anticipation of a CICO scenario, we immediately administered $70 \mathrm{mg}$ rocuronium and planned to start jet ventilation. However, our Monsoon (Acutronic Medical Systems AG, Switzerland) jet ventilator unexpectedly malfunctioned despite being fully operational during the pre-induction check. Face mask ventilation became impossible, and her $\mathrm{SpO}_{2}$ dropped to $70 \%$. The TRIO device was connected to the Ravussin cannula with an oxygen flow of $15 \mathrm{~L} / \mathrm{min}$, and the lungs were insufflated for $4 \mathrm{~s}$ [1]. The lowest $\mathrm{SpO}_{2}$ was $63 \%$, but rapidly reached $100 \%$.

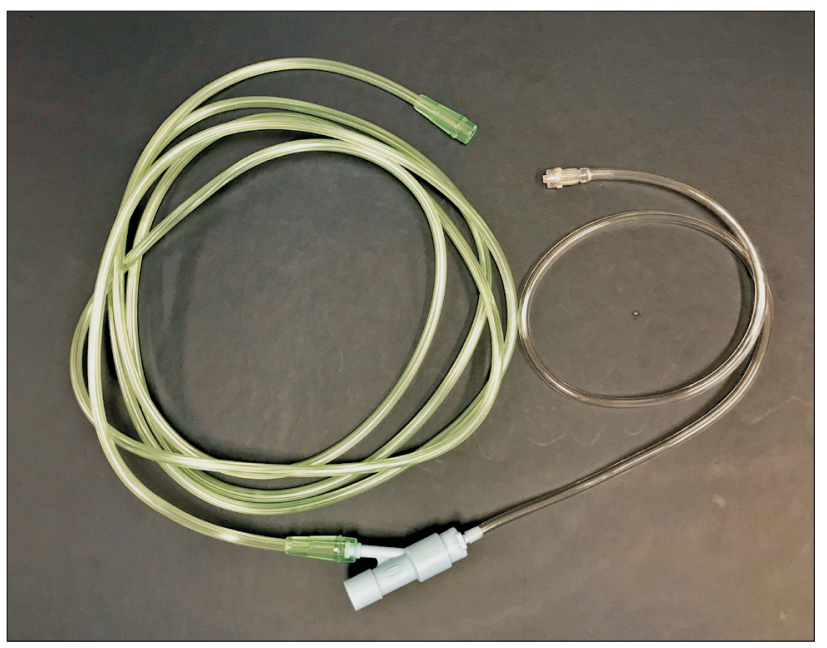

Fig. 2. Trans-tracheal rapid insufflation of oxygen (TRIO) device.

A single attempt at oral Bonfils intubation was performed with simultaneous McCoy laryngoscopy by the most senior consultant anesthetist, but this failed due to distorted anatomy. The ENT surgeons proceeded with an emergency tracheostomy creation which took thirty minutes due to the presence of an undetected goiter. During this time, ten "breaths" of intermittent oxygen insufflation (each lasting $2 \mathrm{~s}$ ) via the TRIO device was administered to keep the $\mathrm{SpO}_{2}$ at $95 \%-100 \%$. Arterial blood gas revealed $\mathrm{pH}$ 6.935, $\mathrm{pCO}_{2} 130 \mathrm{mmHg}$, and $\mathrm{pO}_{2} 136 \mathrm{mmHg}$. We considered converting the Ravussin cannula to a cuffed tube to optimize carbon dioxide clearance, but this was not required as the tracheostomy was completed soon after. Surgery proceeded uneventfully, and a postoperative obstetric review indicated no concerns with regard to the pregnancy. The tracheostomy was decannulated the next day, and the patient was discharged on the second postoperative day.

\section{Discussion}

We accept that some instances of our decision making may be considered controversial, but this report highlights the potential conflict in the process of consent-taking between patient autonomy and the clinician's judgement to provide the best and safest treatment option. In our case, the patient refused an awake tracheostomy, which was strongly conveyed to her as the safest option. We therefore opted to secure her airway after induction of anesthesia, as the consultant was an airway specialist with considerable experience in managing similar airway lesions and agreed with the patient on an alternative and acceptable airway strategy. Plan A was gas induction of anesthesia and Bonfils intubation. Plan B was to maintain oxygenation using high frequency jet ventilation via a needle cricothyroidotomy which would have allowed time for plan C (asleep tracheostomy). 
Our case emphasizes several key points: airway obstruction can worsen rapidly during inhalational induction; rescue of a CICO scenario with a TRIO device; and, the need for backup devices. We discuss the rationale behind our airway strategy.

Awake, flexible, fiberoptic intubation should be avoided in cases of severe airway obstruction, as complete obstruction can result from sedation and airway topicalization [2,3], and from the fiberscope passing through the obstructive lesion, causing a "cork-in-a-bottle" scenario [4]. The next best option was inhalational induction of anesthesia, as it preserves spontaneous respiration. Should complete airway obstruction occur, cessation of the volatile agent will theoretically allow the patient to wake up, although decreased ventilation would increase the time needed [4]. However, the required deep level of anesthesia for intubation precipitated worsening of the airway obstruction in our patient, and face mask ventilation became extremely difficult.

We proceeded on to plan B i.e., oxygenation via needle cricothyroidotomy using high frequency jet ventilation, a temporizing measure to provide time for a more controlled tracheostomy. A pre-induction insertion of a cricothyroidotomy cannula would have been ideal but was not performed because of patient refusal. We chose to use the Monsoon jet ventilator as it has been safely used in cases of upper airway obstruction because it minimizes barotrauma by automatically pausing jet ventilation if the pre-set airway pressure limit ("pause pressure") is exceeded. Unfortunately, in our case, the Monsoon ventilator failed due to a software problem.

After encountering a potential CICO scenario, we paralyzed our patient with rocuronium, with sugammadex readily available to reverse paralysis if required. This is controversial, as it causes loss of muscle tone, ablates spontaneous ventilation, and diminishes the ability to awaken the patient [4]. In severe, fixed, annular tracheo-bronchial obstructive lesions, administering muscle relaxants can improve ventilation through the application of positive pressure ventilation [5]. In contrast, with pedunculated lesions, there is a risk for a ball-valve like obstruction if muscle relaxants are given and positive pressure ventilation is applied [4]. However, the latest difficult airway guidelines recommend the use of muscle relaxants in a CICO scenario, as it may reverse laryngospasm and optimize mask ventilation and laryngoscopy [6]. In addition, it may reduce the risk of negative pressure pulmonary edema caused by forceful spontaneous ventilation in the presence of an obstructed upper airway [7]. Moreover, awakening the patient was not an option because of severe hypoxia, and the higher time needed for sevoflurane redistribution to permit awakening [8].

We salvaged the CICO scenario by rapidly oxygenating the patient using the TRIO device via the needle cricothyroidotomy. Previously known as the "Leroy," this device consists of two pieces of oxygen tubing linked by a thumb operated Y-piece that allows controlled insufflation of the lungs ("breaths") at 15 $\mathrm{L} / \mathrm{min}[1,9]$. The underlying principle is of provision of oxygenation with carbon dioxide clearance being secondary while minimizing the duration and rate of insufflation to reduce the risk of barotrauma [9]. High pressures of up to $4,000 \mathrm{cmH}_{2} \mathrm{O}$ are generated from the oxygen flowmeter, which is directly transferred to the airways, resulting in high intrathoracic pressures in the event of significant or complete upper airway obstruction [10]. Upon obtaining tactile feedback of high pressure via the thumb during Y-piece occlusion, the user can terminate lung insufflation, acting as a safety mechanism against barotrauma. In trials involving live sheep models, Heard et al. established that an initial "breath" of $4 \mathrm{~s}$ delivering 1,000 $\mathrm{ml}$ of oxygen is sufficient to restore oxygenation $[10,11]$. Release of the Y-piece then allows passive expiration of gases. Exhalation of a tidal volume of $500 \mathrm{ml}$ takes at least $30 \mathrm{~s}$ via the $14 \mathrm{G}$ cannula [12]. However, is likely to be longer in case of upper airway obstruction. If more "breaths" are delivered prior to expiration, barotrauma may result. Hence, subsequent "breaths" of $2 \mathrm{~s}$ (delivering 500 $\mathrm{ml}$ oxygen) are delivered when there is inadequate initial $\mathrm{SpO}_{2}$ response or a $\mathrm{SpO}_{2}$ drop of $5 \%$ from the maximal $\mathrm{SpO}_{2}$ attained after the initial insufflation attempt $[10,11]$. The rate of insufflation can be low because oxygen saturation takes at least a minute to fall following an insufflation [11]. Allowing adequate time for lung deflation is more crucial than lung insufflation to minimize barotrauma [13]. The shortest duration for insufflation and longest duration for exhalation should be used.

In contrast, some jet ventilators do not have an expiratory valve, potentially contributing to gas trapping, hypoventilation, and an increased risk of barotrauma in cases of complete airway obstruction [14]. Peak airway pressures are also lower with insufflation devices such as the TRIO, compared to manual high-pressure jet ventilators [15]. However, restoration of normal oxygen saturation may lag behind insufflation of lungs by up to $20 \mathrm{~s}$. As this is primarily an oxygenation device, it should be noted that inadequate ventilation from prolonged expiration and low insufflation rates can also cause hypercarbia and respiratory acidosis. End-tidal capnography is not reliable during TRIO. There have been no studies on the maximum duration of maintaining oxygenation with the TRIO device. However, severe respiratory acidosis has deleterious systemic effects such as cardiovascular instability and increased intra-cranial pressure. As such, the TRIO device should be used as a temporizing measure for oxygenation in a CICO scenario while attempts are made to secure a definitive airway (e.g., tracheostomy) to allow adequate ventilation and oxygenation.

We attempted oral intubation using the rigid Bonfils fiberscope, as it is a slim optical stylet with an anteriorly curved tip that can be manipulated around obstructive peri-glottic lesions, facilitating tracheal intubation. We used it in conjunction with 
laryngoscopy with the McCoy blade as its levering tip lifts the epiglottis and displaces lesions anteriorly, improving the visualization of the glottis. This was not successful, and the surgeons proceeded with the creation of a tracheostomy.

A patient's autonomy may conflict with what is considered the best and safest treatment option in patients with an anticipatedly difficult airway. A logical and stepwise airway plan is therefore required. Alternative equipment should be available and plans in place in case of unexpected equipment failure. The TRIO device is an inexpensive device that can be readily available in every operating theatre to allow for prompt rescue of a CICO scenario.

\section{Conflicts of Interest}

No potential conflict of interest relevant to this article was reported.

\section{Author Contributions}

John Lee (Conceptualization; Writing-original draft; Writingreview \& editing)

Von Vee Ng (Conceptualization; Writing-original draft; Writing-review \& editing)

Constance Teo (Conceptualization; Writing-review \& editing)

Patrick Wong (Conceptualization; Supervision; Writing-original draft; Writing - review \& editing)

\section{ORCID}

John Lee, https://orcid.org/0000-0001-5698-3913

Von Vee Ng, https://orcid.org/0000-0001-8022-3420

Constance Teo, https://orcid.org/0000-0003-4226-2140

Patrick Wong, https://orcid.org/0000-0002-3212-9496

\section{References}

1. Wexler S, Hall K, Chin RY1, Prineas SN. Cannula cricothyroidotomy and rescue oxygenation with the Rapid-O2 ${ }^{\mathrm{TM}}$ oxygen insufflation device in the management of a can't intubate/can't oxygenate scenario. Anaesth Intensive Care 2018; 46: 97-101.

2. Ho AM, Chung DC, Karmakar MK, Gomersall CD, Peng Z, Tay BA. Dynamic airflow limitation after topical anaesthesia of the upper airway. Anaesth Intensive Care 2006; 34: 211-5.

3. Shaw IC, Welchew EA, Harrison BJ, Michael S. Complete airway obstruction during awake fibreoptic intubation. Anaesthesia 1997; 52: 5825 .

4. Wong P, Wong J, Mok MU. Anaesthetic management of acute airway obstruction. Singapore Med J 2016; 57: 110-7.

5. Nouraei SA, Giussani DA, Howard DJ, Sandhu GS, Ferguson C, Patel A. Physiological comparison of spontaneous and positive-pressure ventilation in laryngotracheal stenosis. Br J Anaesth 2008; 101: 419-23.

6. Cook TM, Woodall N, Frerk C. Major complications of airway management in the UK: results of the Fourth National Audit Project of the Royal College of Anaesthetists and the Difficult Airway Society. Part 1: anaesthesia. Br J Anaesth 2011; 106: 617-31.

7. Lemyze M, Mallat J. Understanding negative pressure pulmonary edema. Intensive Care Med 2014; 40: 1140-3.

8. Girgis Y, Frerk CM, Pigott D. Redistribution of halothane and sevoflurane under simulated conditions of acute airway obstruction. Anaesthesia 2001; 56: 613-5.

9. Greenland KB, Bradley WPL, Chapman GA, Goulding G, Irwin MG. Emergency front-of-neck access: scalpel or cannula-and the parable of Buridan’s ass ${ }^{\dagger}$. Br J Anaesth 2017; 118: 811-4.

10. CICO airway management: percutaneous emergency oxygenation techniques, 06 Jet Oxygenation [Internet]. Perth: Royal Perth Hospital; 2013 Dec 16 [cited 2019 Jan 20]. Video 5:28 min. Available from https://www.youtube.com/watch?v=QR7ek7VBNiQ.

11. Heard AM. Percutaneous Emergency Oxygenation Strategies in the "Can't Intubate, Can't Oxygenate" Scenario. Smashwords Edition. Perth, Smashwords, Inc. 2013, pp 1-74.

12. Ryder IG, Paoloni CC, Harle CC. Emergency transtracheal ventilation: assessment of breathing systems chosen by anaesthetists. Anaesthesia 1996; 51: 764-8.

13. Cook TM, Nolan JP, Magee PT, Cranshaw JH. Needle cricothyroidotomy. Anaesthesia 2007; 62: 289-90.

14. Hamaekers AE, Götz T, Borg PA, Enk D. Achieving an adequate minute volume through a $2 \mathrm{~mm}$ transtracheal catheter in simulated upper airway obstruction using a modified industrial ejector. Br J Anaesth 2010; 104: 382-6.

15. Berry M, Tzeng Y, Marsland C. Percutaneous transtracheal ventilation in an obstructed airway model in post-apnoeic sheep. Br J Anaesth 2014; 113: 1039-45. 